\title{
PAPER
}

CrossMark

OPEN ACCESS

RECEIVED

9 November 2020

REVISED

7 April 2021

ACCEPTED FOR PUBLICATION

13 April 2021

PUBLISHED

10 May 2021

Original content from thi

work may be used under

the terms of the Creative

Commons Attribution 4.0

licence.

Any further distribution of this work must maintain

attribution to the

author(s) and the title of

the work, journal citation

and DOI.

\section{Particle tracking and beam optics analysis on a toroidal gantry for proton therapy}

\author{
E Felcini $^{1,2, *}\left(\mathbb{\infty}\right.$, L Bottura $^{1}$, A Gerbershagen $^{3}, \mathrm{~J} \mathrm{van} \mathrm{Nugteren}^{1}$ and B Dutoit ${ }^{2}$ \\ 1 TE Department-CERN, 1211 Geneva 23, Switzerland \\ 2 Groupe SCI IC BD-EPFL, 1015, Lausanne, Switzerland \\ 3 EN Department-CERN, 1211 Geneva 23, Switzerland \\ * Author to whom any correspondence should be addressed. \\ E-mail: enrico.felcini@cern.ch \\ Keywords: particle tracking, beam optics, toroidal magnets, proton therapy gantry
}

\begin{abstract}
GaToroid is a concept of toroidal gantry for hadron therapy under investigation at CERN It makes use of the toroidal magnetic field between each pair of coils to steer and focus the particle beams down to the patient. This peculiar concept requires detailed studies on particle tracking and beam optics to optimise the winding geometry and explore the properties of the system. The work presented in this manuscript is focused on the features of a GaToroid system for protons, specifically designed to minimise the footprint and weight of the gantry. Firstly, a two-dimensional single particle tracking was developed to optimise the coil geometry and the toroidal magnetic field, aiming to the maximisation of the energy acceptance of the magnet. Particles over the whole spectrum of treatment energy are directed at isocenter within $1 \mathrm{~mm}$ of precision. This procedure, restricted to the symmetry plane between each pair of coils, defines different beam orbits, function of the beam energy. Subsequently, a three-dimensional particle tracking was implemented to evaluate the interaction of a beam of finite dimensions with the complete magnetic field map in vacuum. The parameters of the simulated beam at the isocenter are coherent with the clinical requirements. The results of the threedimensional tracking were then used to calculate the linear transfer matrix associated to each beam orbit. Finally, the option of performing the beam spot scanning at the isocenter by acting on the upstream steering magnet has been investigated, highlighting the potential of the concept, as well as the limitations related to the scanning field dimension and source-to-axis distance. In conclusion, the results described in this paper represent a crucial step toward the understanding of the beam optics properties of a GaToroid gantry.
\end{abstract}

\section{Introduction}

Hadron therapy is considered one of the most advanced and effective cancer treatment options based on radiation. Profiting from the sharp energy deposition, i.e. the Bragg peak typical of protons and heavy ions, it is possible to deliver a well-localised dosage of ionising radiation to the tumour cells, sparing healthy tissues from a large share of detrimental dose (Kraft 1990, Tsujii et al 2007, 2013, Durante and Orecchia 2017, Takada 2020). The downsides of this technology are the complexity, size and cost of the structures required to accelerate and direct particle beams down to the patient, namely accelerators and gantries. This is one of the main reasons why the diffusion of hadron therapy centres has been limited (Schippers et al 2018). At the same time, the potential of growth and societal impact are high motivators to study new concepts and techniques to deliver beams suitable for therapy using simpler, smaller and cost-effective machines. This is especially true for heavy ions therapy, where the market penetration is still superficial and the margin for technological improvement and cost reduction is much larger (Yan et al 2016). Different accelerator configurations are commercially available or are under study, ranging from cyclotrons to synchrotrons and linacs (Farr et al 2018, Myers et al 2019). At the same 


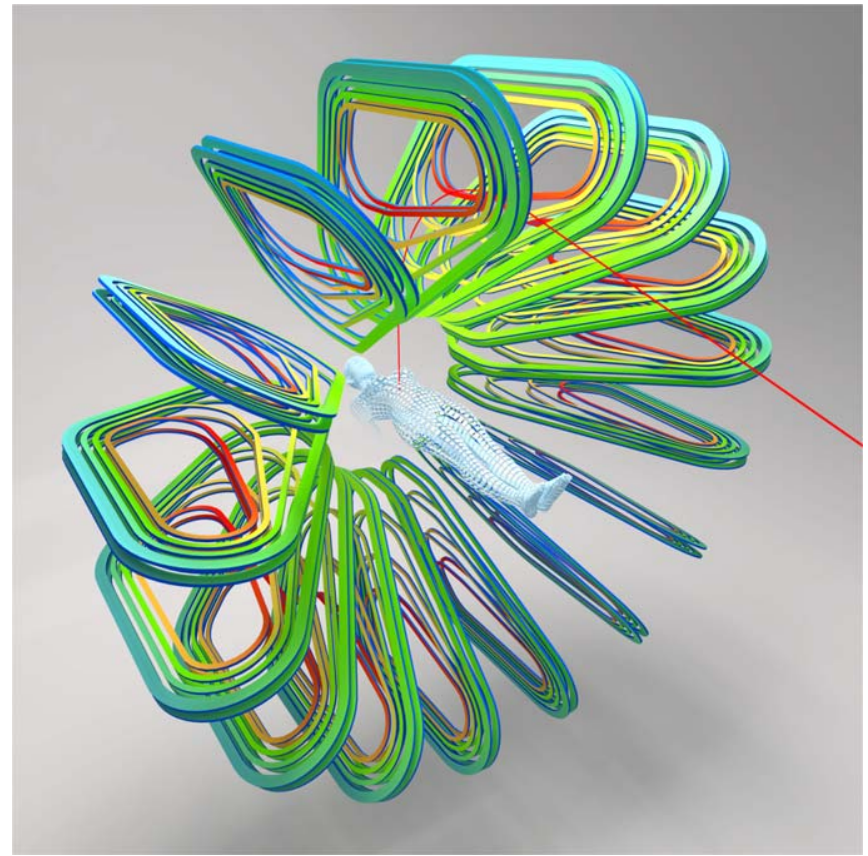

Figure 1. Artistic illustration of the GaToroid working principle, where the patient plays in the torus bore, along the torus axis, and the space available between each pair of coils can be used to irradiate from multiple directions.

time, several gantries for proton therapy are available on the market and currently in operation. These commercial gantry solutions range from 7.2 to $10.4 \mathrm{~m}$ in diameter and from 110 tons to 270 tons in weight (Von Essen et al 1982, Pedroni et al 2011, Kumata 2013, Pearson et al 2014, Hadron et al 2014, Umezawa 2014, Koschik et al 2015, Symonds et al 2019, La Civita 2019). Regarding heavier ions, the complexity of the machines has prevented the establishment of a real market, and, to the present day, only two facilities in the world can operate with gantries for ions (Fuchs and Weinrich 2004, Iwata et al 2012). A wide range of configuration has been proposed with the final goal of reducing the weight and size of the installations, both for proton and heavy ions (Trbojevic et al 2007, Pullia et al 2009, Robin et al 2011, Wan et al 2015, Gerbershagen et al 2016, Iwata et al 2016, Bromberg and Michael 2017, Masood et al 2017, Kim and Yoon 2019, Kang and Pang 2020, Nesteruk et al 2021). The concept of the toroidal gantry, GaToroid (Bottura 2018, Bottura et al 2020, Felcini 2021), can be positioned among these initiatives. On the one hand, to reduce the size of the machine, GaToroid is conceived to use a set of high-field superconducting coils operating in steady-state. On the other hand, to simplify the mechanics and lessen the overall weight, this concept allows directing the beam to the patient from a discrete number of directions avoiding, in principle, any rotation of the components.

In this paper, we provide for clarity a much-simplified description of the working principle. The reader will find more details in the above references.

Although such a gantry concept is very appealing, several issues and challenges need to be addressed. Besides the design of superconducting coils (Felcini et al 2020) and their prototyping (Felcini et al 2021), the detailed beam-optics in the toroidal field of GaToroid is one of the crucial topics for the advancement of this project. Examples of particle tracking in toroidal lens are reported in Mohri et al (1977), Von Essen et al (1982), Andreev and Yudin (1998). Somewhat related to beam optics and tracking is the expansion in series of the toroidal field. Harmonics expansion of toroidal magnetic field are described by van Milligen and Lopez Fraguas (1994), and numerically developed in Brouwer et al (2013), Gambini et al (2020). However, to our knowledge, there is no theory that can support the analysis of the beam optics features of a toroidal gantry for hadron therapy. This is the main driver for this paper, namely to present a first analysis of beam transmission in a toroidal field to set the basis for the understanding and the construction of GaToroid. After a brief explanation of the working principle and configuration of GaToroid, we describe the method used for the analysis of beam transmission, based on two- and three-dimensional (2D and 3D) tracking, and the reduction of results to an equivalent transfer matrix. We then show the results of the application of this method to GaToroid, and in particular (i) how to minimise the distance between the focal points of different energies at isocenter, (ii) how to match beam properties to obtain the required beam size at the isocenter, and (iii) how to scan a given area around the isocenter. For the sake of clarity and simplicity, the studies reported in this manuscript are focused and limited to the proton GaToroid configuration described in Felcini et al (2020), Felcini (2021). This configuration is composed of 16 coils symmetrically arranged around the toroidal axis. In comparison with the aforementioned commercial 
solutions for protons, it allows a reduction of the machine size by a factor of 2 , with an outer diameter of $3.3 \mathrm{~m}$ and an internal bore of $0.8 \mathrm{~m}$. At the same time, the weight can be reduced by one order of magnitude, with an overall mechanical structure of about 12 tons. Other configurations, based on different ion species and topologies, are being investigated with identical methodology (Felcini 2021), but are not reported in this manuscript.

\section{GaToroid working principle}

GaToroid is a toroidal gantry concept presently in development. The basic idea is to direct particles to a tumour location using a steady-state toroidal magnetic field generated by $N_{c}$ appropriately shaped coils arranged around the $\mathbb{Z}$-axis of the torus, along which the patient is positioned (Bottura et al 2020). The electro-magnetic design of the coil geometry results in a steady-state toroidal magnetic field with very large beam acceptance, i.e. able to bend particle beams in the whole spectrum of treatment energies. A representation of the working principle is presented in figure 1 , where a schematic beam trajectory is highlighted in red.

An upstream dipole, the vector magnet, is used to steer the beam in between the $N_{c}$ coils, providing $N_{c}$ discrete azimuthal directions of irradiation around the patient. The space in-between each pair of coils is used for beam transport, namely at azimuthal angles $\theta_{i}=i 2 \pi / N_{c}$ with $i=1 \ldots N_{c}$. The same vector magnet is used to deviate the beam with a suitable polar angle $\alpha_{E}$, function of the beam kinetic energy, modifying the radial position at the entry of the torus so as to achieve consistent output at the isocenter. The vector magnet is positioned along the axis at $\mathbb{Z}=-z_{V}$, and if the distance from the torus is sufficiently large, the polar angle dependency on beam energy can be approximated as:

$$
\alpha_{E} \approx \frac{R_{\text {in }}+\rho_{E}}{z_{V}-\rho_{E}},
$$

where $R_{\text {in }}$ is the internal radius of the torus and $\rho_{E}$ is the radius of curvature associated to the beam rigidity $(B \rho)$ and the ideal uniform magnetic field $B_{0}$, i.e. $\rho_{E}=(B \rho) / B_{0}$. The exact expression of $\alpha_{E}$ is reported in Bottura et al (2020). For the configuration described in this paper, the polar angle ranges from $13^{\circ}$ at $70 \mathrm{MeV}$ to $20^{\circ}$ at $250 \mathrm{MeV}$.

At the same time, as detailed explain later in the text, a deviation from the nominal polar and azimuthal angles of a few tenths of degrees can be used to perform a pencil beam scan around the isocenter. The accuracy required at the vector for this operation is in the order of $0.01^{\circ}$.

The coupling of these different features, selection of the irradiation direction $\left(\theta_{i}\right)$, adjustment as a function of energy $\left(\alpha_{E}\right)$ and pencil beam scanning ( $\delta \theta$ and $\delta \alpha$ ), introduces important complexities in the design of the vector magnet. The different orders of magnitude of the required angles, suggests the division of the vector magnet into two different elements, dedicated to large and small deflections respectively.

The first and most important of these elements is the magnet responsible for the selection of the direction of irradiation $\left(\theta_{i}\right)$ and polar angle as function beam of energy $\left(\alpha_{E}\right)$. We will refer to it as the angle-selector. This magnet can be curved and installed on a rotating structure, which would be the only rotating part of the installation, reducing the required range of $\alpha_{E}$ to $7^{\circ}\left(13^{\circ}\right.$ at $70 \mathrm{MeV}$ to $20^{\circ}$ at $\left.250 \mathrm{MeV}\right)$ in a rectangular aperture.

Considering instead a straight and non-rotating magnet, the use of a horizontal-vertical combined dipole would be an elegant solution. However, it would require a large square aperture, with an angular extension of $\pm 20^{\circ}$ in both planes. In this case, a superconducting option (field greater than $3 \mathrm{~T}$ ) could help to reduce the length and aperture of the magnet but would introduce limitations in the ramping speed and change of beam direction.

The second magnetic element is dedicated to pencil beam scanning and it is equivalent in principle to a traditional scanning system. If the angle-selector is characterised by a rectangular aperture, to avoid increasing the aperture in both planes it can be convenient to place a first scanning magnet upstream and a second one downstream.

Given the instrumental role of the vector magnet in the whole GaToroid project, we are currently evaluating and analysing these options, aiming to the best compromise between footprint, scanning speed and complexity.

\section{Material and methods}

As anticipated above, the focus of this paper is the particle tracking and beam optics for the GaToroid magnets. We approach the problem using first a simplified 2D particle tracking, which has the benefit of speed and simplicity, and it is used for the optimisation of the toroid geometry. The method is then extended to a 3D tracking in the complete field map of the torus, aiming at defining and understanding the beam parameters along the full envelope of trajectories. We describe below the field mapping and tracking methods, in 2D and 3D, 


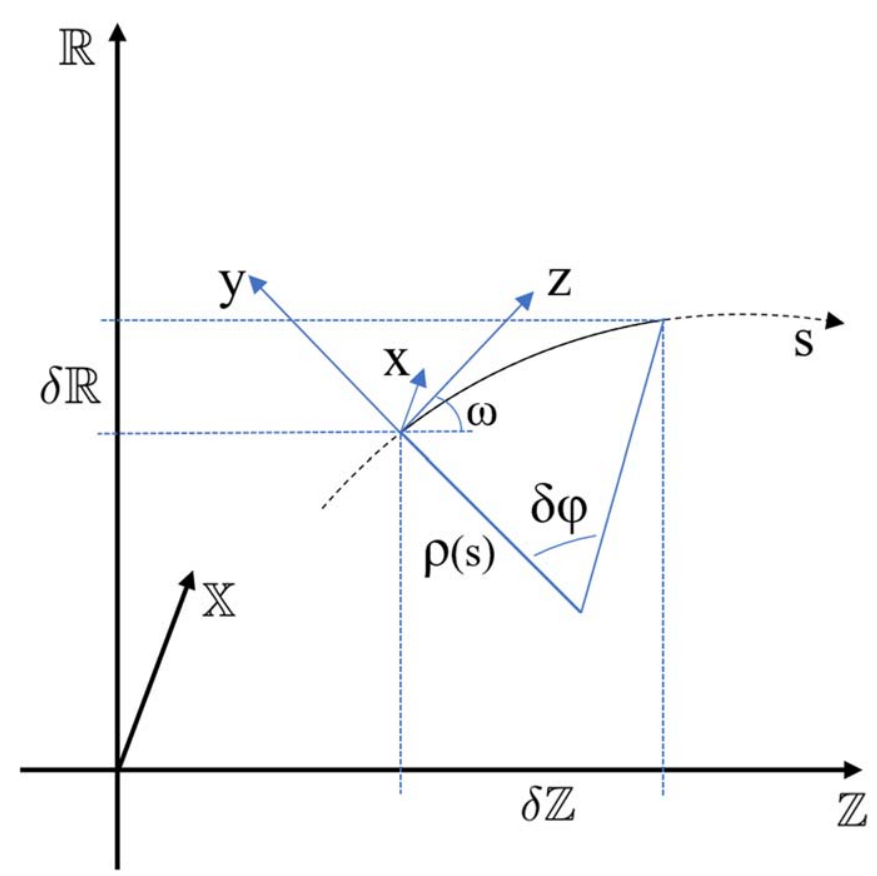

Figure 2. Representation of the global reference system $(z, y)$ and co-moving coordinate system $(z, y)$ along the orbits.

the seed generation for 3D tracking, and the procedure followed to generate a beam transfer matrix from 3D tracking simulations.

\section{Field calculation method}

The complete three-dimensional layout of the coils and the magnetic field calculation are performed with Field 2017 (van Nugteren 2011, 2016) (the software is now developed under the name of RAT Van Nugteren 2019).

The coil geometry is described using line elements of $1 \mathrm{~mm}$ length. In the cross-section of each grade, i.e. planar subsoil, 15 line elements are considered. The software integrates the magnetic field contribution of the line elements at the required target points. In the context of this paper, two methods were used for such a computation: direct Biot-Savart and multi-level fast multipole method (MLFMM) (Greengard 1987, Beatson and Greengar).

The first method integrates the contribution of $\mathrm{N}$ source line elements for each target point. For a set of $\mathrm{M}$ target points, the computational complexity of this method scales as $\mathrm{O}(\mathrm{NxM})$. To reduce the complexity to $\mathrm{O}(\mathrm{N}+\mathrm{M})$ (linear scaling), the MLFMM method can be used to approximate the field contribution of the line elements with a spherical harmonic expansion. Although the MLFMM method generates an approximated solution, the calculations are between one and two orders of magnitude faster.

For this reason, the MLFMM method was used to calculate the field in combination with the coil geometry optimisation and two-dimensional particle tracking that require a large number of iterations. The direct BiotSavart method was employed to refine the results of the optimisation as well as to calculate an accurate threedimensional field map used to investigate the beam optics properties of the system.

The problem is linear since no iron is considered in the system and the diamagnetic effects in the superconductors are neglected.

For the two-dimensional particle tracking, we calculated the magnetic field map on the symmetry plane between a pair of coils, i.e. at the azimuthal angle $\theta_{i}$, using a grid of $3 \mathrm{~mm}$ square elements.

For the three-dimensional particle tracking, the volume between a pair of coils, i.e. around the azimuthal angle $\theta_{i}$, is described with a cubic mesh of $3 \mathrm{~mm}$ edges.

For both methods, we used a linear interpolation of the magnetic field during the integration of the particle trajectories. We cannot appreciate relevant differences in the results using an interpolation based on a cubic function. 


\section{Particle tracking algorithms (2D and 3D)}

The motion of a charged particle subject to a magnetic field is governed by the Lorentz force and is described by:

$$
F_{L}=m \frac{d v}{d t}=q(v \times B),
$$

where $v, q$ and $m$ are respectively velocity, charge and relativist mass $\left(m_{0} \gamma\right)$ of the particle, and B is the magnetic field (Lawson et al 1984). Above we have neglected the effects of external and beam-generated electric fields, i.e. space charge and wakefields, which are not relevant to our analysis (Ferrario et al 2013). The solution of equation (2) uniquely determines the trajectory of the particles in vacuum.

\section{Two-dimensional particle tracking}

For the numerical solution of the beam trajectory in 2D, it is convenient to introduce the concept of beam rigidity as follows. We consider a homogeneous magnetic field, with one component only in $x$ direction, and a particle motion perpendicular to the field, i.e. in the $(z, y)$ plane. In this simple case, the particle orbit is circular, with a radius of curvature $\rho$. On this orbit the particle experiences a centripetal force:

$$
F_{c e n t r}=\frac{m v^{2}}{\rho} .
$$

The beam rigidity can be obtained combining equations (2) and (3):

$$
(B \rho)=\frac{m v}{q} .
$$

We see that once the beam rigidity, i.e. the momentum-to-charge ratio of a particle, and the value of the magnetic field transverse to the particle velocity are known, it is possible to compute the radius of the circular orbit.

Our tracking algorithm is based on the simple principle above, introducing a local coordinate system moving with the particle, as shown in figure 2. At an arbitrary position $s$ the coordinate system has the $z$-axis tangent to the beam velocity $v$, the $x$-axis aligned with the component of the local field vector normal to $s$, indicated as $B_{x}(s)$, and the $y$-axis normal to both $z$ and $x$.

The bending radius, $\rho(s)$, in the $(y, z)$ plane at a point $s$ along the particle orbit is:

$$
\rho(s)=\frac{(B \rho)}{B_{x}(s)} .
$$

One particular case is when particles travel in the $(\mathbb{Z}, \mathbb{R})$ plane located between each pair of coils, i.e. $\theta_{i}$, where the toroidal magnetic field is orthogonal to the plane. In this case, the particle tracking reduces to $2 \mathrm{D}$, and can be considerably simplified. With a change of coordinate, we can derive the infinitesimal variations in the position of the particle in the global reference system $(\mathbb{Z}, \mathbb{R})$ as follows:

$$
\begin{array}{r}
\delta \mathbb{Z}=\sin (\omega) \rho(s)(1-\cos (\delta \varphi))+\cos (\omega) \rho(s) \sin (\delta \varphi) \\
\delta \mathbb{R}=-\cos (\omega) \rho(s)(1-\cos (\delta \varphi))+\sin (\omega) \rho(s) \sin (\delta \varphi),
\end{array}
$$

where $\omega$ is the angle between the moving and the global coordinate system, (between $z$ and $\mathbb{Z}$ ), and $\delta \varphi$ is the angle of the arc formed by the orbit along the differential distance $\delta s,(\delta \varphi=\delta s / \rho(s))$. The above equations are integrated using a forward Euler algorithm with a step $\delta s=0.1 \mathrm{~mm}$ to obtain the particle trajectories in 2D.

If the three-dimensional particle tracking described in the next section requires hundreds of seconds to describe the trajectory of particles from the vector magnets to the isocenter, this simplified two-dimensional tracking can reduce the computational time down to few seconds. For this reason, we used this simple and computationally light algorithm for an iterative optimisation of the geometry of the coils, based on the result of particle tracking, as described later.

\section{Three-dimensional particle tracking}

Considering a particle beam of finite transverse dimensions, the interaction between the particles and magnetic field can be no longer limited to the symmetry plane between coils. Therefore, a complete solution of the threedimensional equation of motion of charged particles in a magnetic field, described by equation (2), is required. As presented in figure 2 along each orbit s, derived from the two-dimensional tracking, it is possible to define the co-moving transverse plane $(x-y)$, where classical transverse beam dynamic theory can be applied. Around each orbit s the properties of the beam and the particle distribution are defined.

The solution in three-dimensions of equation (2) is performed through a six-stage, fifth-order, Runge-Kutta method (MATLAB ode 45 Yang et al), with a relative error tolerance of $10^{-6}$ and an initial time step of $10^{-8}$.

The magnetic field is obtained from a linear interpolation on the calculation grid, as described earlier. Finally, all tracking runs were done considering a pure transverse beam (see also later about beam seed 
generation), neglecting any longitudinal effect. Furthermore, the method described does not take into account any particle interaction with matter, assuming vacuum conditions down to the patient. The study of beam interactions with matter is beyond the scope of this paper, and we refer to previous works (Garonna et al 2018) for the multiple scattering effects on the beam travelling through ionisation chambers, air gaps and patient equivalent tissues that can be estimated through dedicated MonteCarlo simulations.

\section{Beam seed}

A key input to particle tracking in 3D is the seed, i.e. the distribution of initial particle position and velocity in the transverse plane $(x-y)$, which needs to be representative of relevant beam parameters. To generate the seed for the $3 \mathrm{D}$ tracking we resort to classical transverse beam dynamic theory.

In particle accelerators it is common practice to define the beam properties trough the Twiss parameters, $\alpha$ and $\beta$ (Lawson et al 1984):

$$
E(s)=\sqrt{\varepsilon \beta(s)} \quad \alpha(s) \equiv-\frac{1}{2} \beta(s)^{\prime}
$$

where $E(s)$ indicates the particles oscillation range along the orbit $s$, and $\varepsilon$ the emittance, considered as an invariant of the system. The parameter $\beta$ is related to the beam size and $\alpha$ to its first derivative, i.e. the tendency of the beam to converge or diverge.

We considered a single plane, $x$, to explain the method. To generate a seed with desired beam parameters, we start from uncorrelated Gaussian distributions of particle positions, $x_{u c}$, and derivatives, $x_{u c}{ }^{\prime}$. We then apply a Cholesky transformation to obtain the proper correlation between particles position and velocity (Lord et al 2007, Kyng and Konstandatos 2014). For a given particle distribution, the covariance matrix $\sum$ can be written as:

$$
\Sigma=\left[\begin{array}{cc}
\left\langle x^{2}\right\rangle & \left\langle x x^{\prime}\right\rangle \\
\left\langle x x^{\prime}\right\rangle & \left\langle x^{\prime 2}\right\rangle
\end{array}\right]=\varepsilon\left[\begin{array}{cc}
\beta & -\alpha \\
-\alpha & \gamma
\end{array}\right]
$$

with $\gamma=\left(1+\alpha^{2}\right) /(\beta)$, and the emittance given by $\varepsilon=\sqrt{(\operatorname{det} \Sigma)}$.

The covariance matrix $\sum$ is decomposed using a Cholesky decomposition using the lower-triangular matrix $L$, or:

$$
\Sigma=L L^{T}
$$

Finally, the sample vector of uncorrelated position and derivatives is multiplied by the $L$ matrix, yielding a vector of position and derivatives with the covariance properties of the system being modelled:

$$
\left[\begin{array}{c}
x \\
x^{\prime}
\end{array}\right]=L\left[\begin{array}{c}
x_{u c} \\
x_{u c}^{\prime}
\end{array}\right]
$$

Using this method, we translate the Twiss parameters into particle position and velocity distributions. The same approach is applied to $y-y^{\prime}$ phase space to model the beam parameters on the other plane.

An advantage of this method is that we can use equation (8) to verify that the values of $\alpha$ and $\beta$ after the Cholesky transformation are the desired ones. Discrepancies below $0.5 \%$ are observed simulating at least 100000 particles. This kind of error is considered acceptable and the presented simulations are performed considering random normal distributions on position and velocity of 100000 particles on both planes.

A last practical step is to project the correlated particle distribution so that the initial average orbit matches the ideal beam trajectory. Position and velocity vectors are rotated by the angle $\omega$ around the $\mathbb{X}$ axis (see figure 2). The relation between local and global reference systems at the beginning of the tracking algorithm $\left(\mathbb{Z}=z_{V}\right.$ and $\left.\omega=\alpha_{E}\right)$ are:

$$
\begin{aligned}
& \mathbb{X}=x \\
& \mathbb{Z}=y \cos \alpha_{E}-z \sin \alpha_{E} \\
& \mathbb{R}=y \sin \alpha_{E}+z \cos \alpha_{E} \\
& v_{\mathbb{X}}=v_{p} \sin x^{\prime} \\
& v_{\mathbb{Z}}=v_{p}\left(\cos y^{\prime} \cos \alpha_{E}-\sin y^{\prime} \sin \alpha_{E}\right) \cos x^{\prime} \\
& v_{\mathbb{R}}=v_{p}\left(\cos y^{\prime} \sin \alpha_{E}+\sin y^{\prime} \cos \alpha_{E}\right) \cos x^{\prime},
\end{aligned}
$$

where $v_{p}$ is the particle velocity calculated using relativistic correction:

$$
v_{p}=c \sqrt{1-\frac{1}{\gamma_{L}^{2}}}
$$

and $\gamma_{L}$ is the Lorentz factor, defined as the ratio between total energy of the particle and its rest energy. 


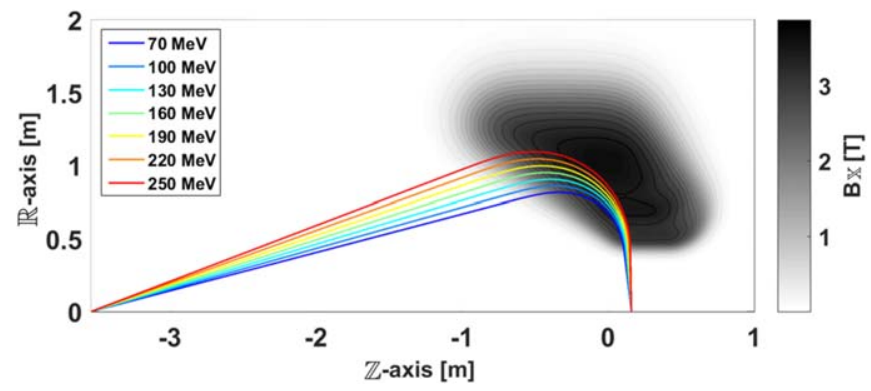

Figure 3. Two-dimensional particle tracking for the optimised coil geometry, on top of the magnetic field map (grey scale).

Finally, it is possible to introduce an arbitrary momentum spread, $d P / P$, in the beam. To implement this feature, we impose a Gaussian random distribution in the particle velocity $v_{p}$.

\section{Linear transfer matrix}

From the beam dynamics point of view, magnetic elements, such as dipoles and quadrupoles, are usually represented with linear transfer matrices. This simple, yet powerful, description of magnets allows evaluating the main beam parameters, i.e. $\alpha$ and $\beta$, with sequences of matrix multiplications, avoiding more complex and time consuming tracking simulations. Ideally, it would be very advantageous to represent the GaToroid system through a linear transfer matrix. This fast and practical tool could then be used for iterative matching the torus to upstream magnets and accelerators.

To achieve this, we model the GaToroid system as $\mathrm{X}_{1}=\mathbf{M X _ { 0 }}$, where $\mathrm{X}_{1}$ and $\mathrm{X}_{0}$ are position and angular vectors at the isocenter and at the vector magnet location respectively:

$$
\left[\begin{array}{c}
x \\
x^{\prime} \\
y \\
y^{\prime}
\end{array}\right]_{1}=\left[\begin{array}{llll}
m_{11} & m_{12} & m_{13} & m_{14} \\
m_{21} & m_{22} & m_{23} & m_{24} \\
m_{31} & m_{32} & m_{33} & m_{34} \\
m_{41} & m_{42} & m_{43} & m_{44}
\end{array}\right]\left[\begin{array}{c}
x \\
x^{\prime} \\
y \\
y^{\prime}
\end{array}\right]_{0}
$$

$\mathrm{X}_{0}$ is the vector of input seed (at the exit of the vector magnet), while $\mathrm{X}_{1}$ is the result of the $3 \mathrm{D}$ particle tracking (at the isocenter). The transfer matrix $\mathbf{M}$ can be calculated as:

$$
\boldsymbol{M}=\boldsymbol{X}_{1} \boldsymbol{X}_{0}^{T}\left(\boldsymbol{X}_{0} \boldsymbol{X}_{0}^{T}\right)^{-1}
$$

Note that each beam energy defines its own orbit and, therefore, at each beam energy the transfer matrix needs to be evaluated. To verify the accuracy of this solution, the tracked particles at isocenter, expressed as $\mathrm{X}_{1}$, have been compared with the particles calculated through the transfer matrix, $\mathrm{X}_{1 \mathrm{M}}=\mathrm{MX}_{0}$. The relative error is defined as:

$$
\lambda=\frac{\sigma\left(X_{1 M}\right)-\sigma\left(X_{1}\right)}{\sigma\left(X_{1}\right)}
$$

where $\sigma$ represents the standard deviation of the particle distributions in $x, x^{\prime}, y$ and $y^{\prime}$. The parameter $\lambda$ provides an estimation of the accuracy of the beam parameters calculated with tracking and transfer matrices. It has to be intended as a global error on the beam, not on single particles. Finally, to prove the physic consistency of the calculated matrices, it is important to verify the value of their determinants is equal to one, as a consequence of the Liouville's theorem (Lawson et al 1984).

\section{Results and discussion}

\section{D particle tracking}

We used the 2D tracking method described earlier to calculate trajectories at different kinetic energies, i.e. for protons $70-250 \mathrm{MeV}$, coupled with an iterative optimisation of the coils geometry.

The winding geometry was parametrised through the vector magnet position $z_{V}$, internal bore radius $R_{\text {in }}$ and ideal magnetic flux density $B_{0}$, while the current distribution was modified subdividing the coils into 5 grades, i.e. planar sub-coils composing the winding, and adjusting their relative radial distance (Bottura et al 2020, Felcini et al 2020, Felcini 2021). The optimisation algorithm was designed to iterate on 7 free parameters, describing the coil geometry and current distribution.

Beam tracking in the specified energy range was run for each coil geometry, and the coil geometry was modified to minimise the distance between the focal points of different energies at the isocenter in the whole 

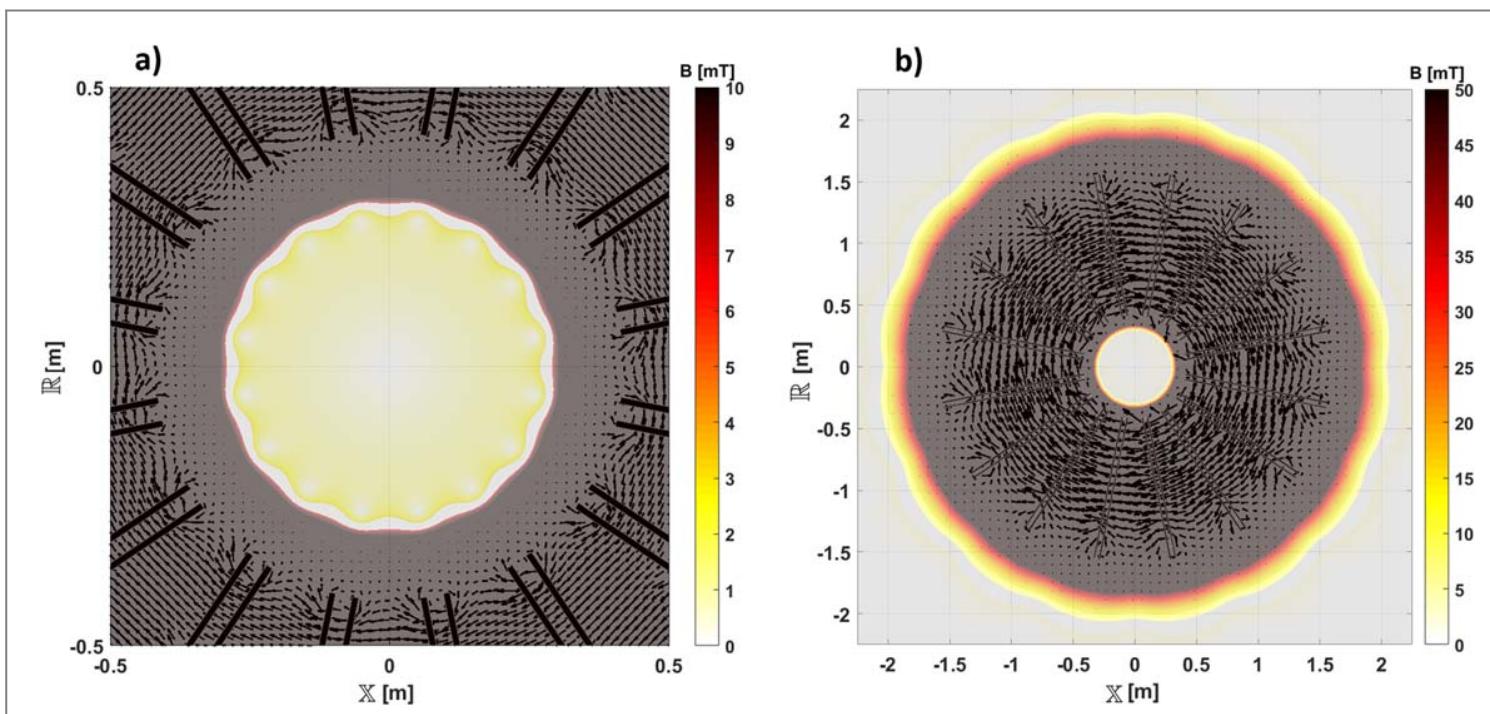

Figure 4. Detail of the magnetic field in the GaToroid bore region (a) and outside the whole torus (b). The colour map indicates values up to $10 \mathrm{mT}$ (a) and $50 \mathrm{mT}$ (b); the grey area of the bore has a flux density greater than $10 \mathrm{mT}$ (a) and $50 \mathrm{mT}$ (b).

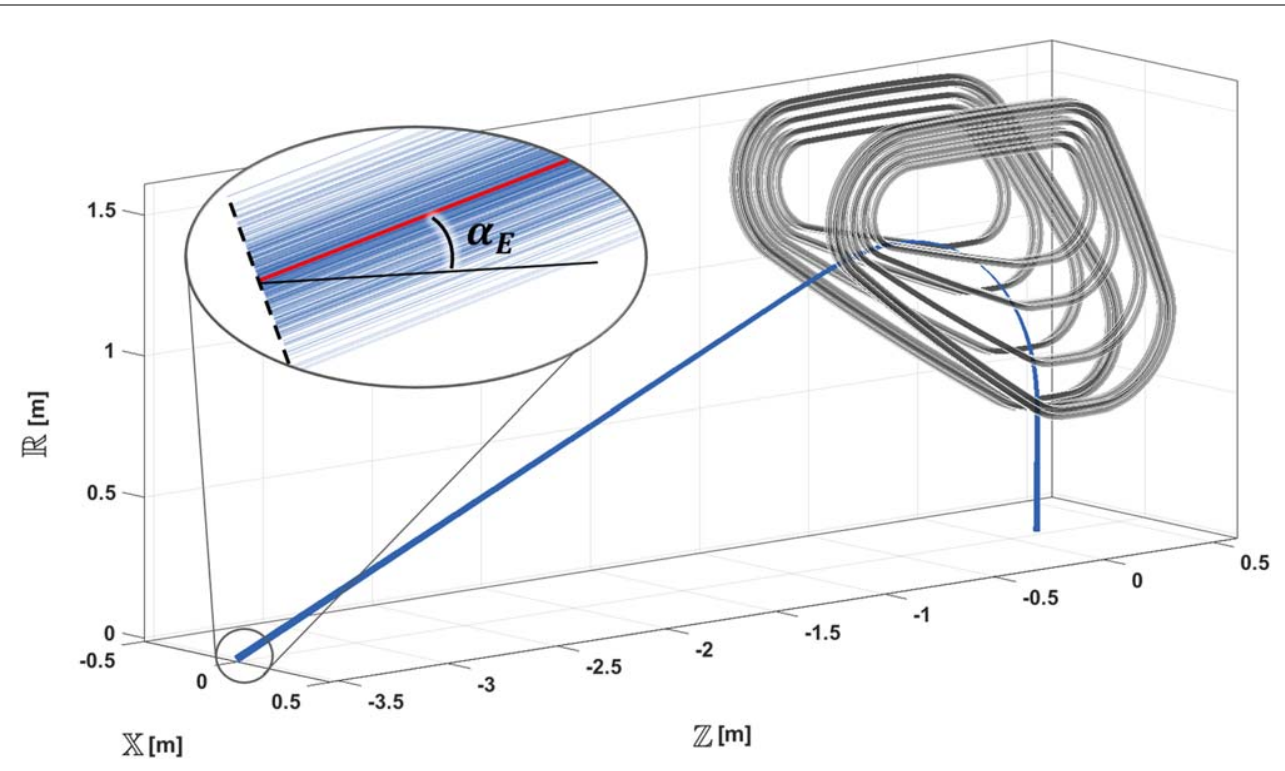

Figure 5. Particle trajectories for a $250 \mathrm{MeV}$ beam described in table 1 together with two coils of the torus. A zoom view at $\mathbb{Z}=-z_{V}$ shows the reference orbit (in red) and the reference plane (dashed line).

treatment spectrum. The demand on the beam requirements at the patient location was implemented as a weighted minimisation function including position and angle with adjustable parameters. For the results reported here, the algorithm was run to reduce the gap between beams position at the isocenter, even if this implies varying the angle of incidence.

The results of the 2D particle tracking optimisation are presented in figure 3 for the complete energy range of proton treatments, i.e. $70-250 \mathrm{MeV}$, together with a magnetic field map produced by the whole torus in the symmetry plane between two coils. The detailed description of the GaToroid magnetic design is presented in Felcini (2021). The convergence at isocenter is within $1 \mathrm{~mm}$, with a maximum angle difference between the orbits of about $5^{\circ}$.

Concerning the field map, it is interesting to note that the magnetic field value at the isocenter is nearly zero. Due to the axisymmetric configuration, the magnetic field at the isocentre ( $\mathbb{X}=0$ and $\mathbb{R}=0$ ), is below the critical threshold for instrumentation and humans safety (order of $\mu \mathrm{T}$ ), as shown in figure 4(a). The field rapidly decays with the radius inside the bore and at $\mathbb{R}=30 \mathrm{~cm}$ is in the order of $10 \mathrm{mT}$. In figure $4(\mathrm{a})$, values above 10 $\mathrm{mT}$ are indicated in grey. The magnetic field map inside the GaToroid bore may create interesting options for the integration of beam and range monitor devices, as described in Bottura et al (2020). 
Table 1.250 MeV beam input and output parameters.

\begin{tabular}{lllll}
\hline Parameter & Unit & Input & $\begin{array}{l}\text { Output } \\
(250 \mathrm{MeV})\end{array}$ & $\begin{array}{l}\text { Output } \\
(70 \mathrm{MeV})\end{array}$ \\
\hline$N_{\text {particles }}$ & & $10^{5}$ & $10^{5}$ & $10^{5}$ \\
$\Delta P / P$ & & $0 \%$ & $0 \%$ & $0 \%$ \\
$\varepsilon_{x-1 \sigma}$ & $(\mathrm{mm}$ mrad $)$ & 1 & 1 & 1 \\
$\varepsilon_{y-1 \sigma}$ & $(\mathrm{mm}$ mrad $)$ & 1 & 1 & 1 \\
$\alpha_{x}$ & & 9.5 & -2.9 & -60.8 \\
$\beta_{x}$ & $(\mathrm{~m})$ & 35.0 & 4.5 & 47.5 \\
$\alpha_{y}$ & & 4.8 & -2.4 & -1.5 \\
$\beta_{y}$ & $(\mathrm{~m})$ & 20.0 & 4.1 & 2.7 \\
\hline
\end{tabular}
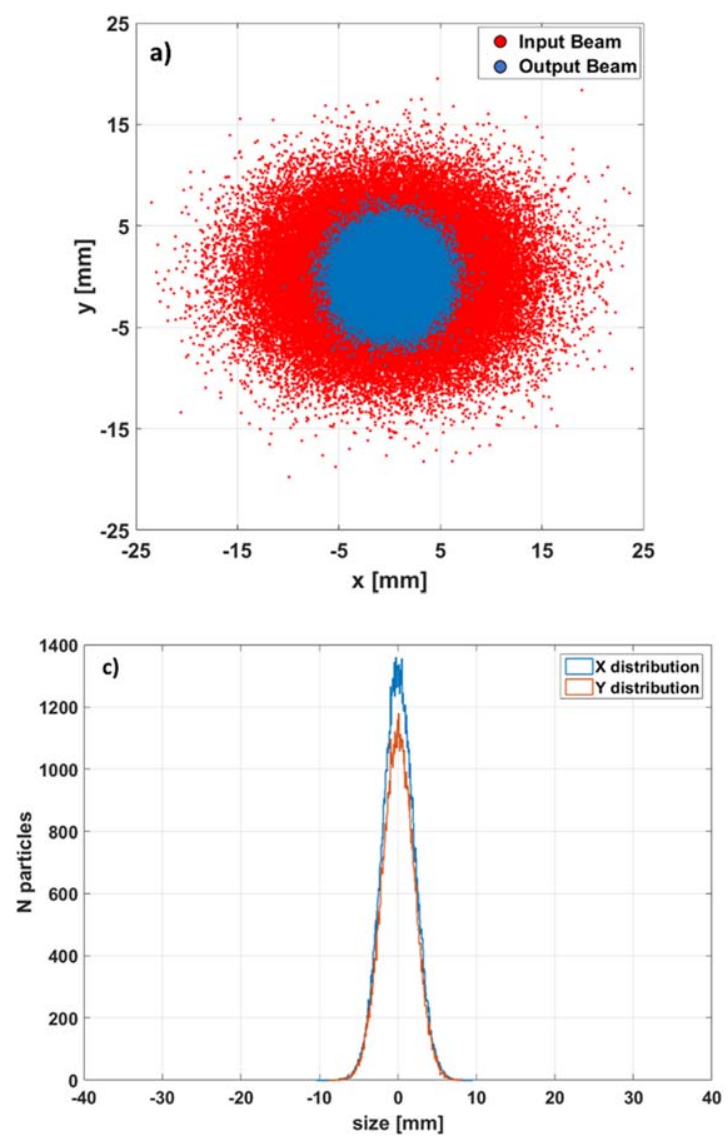
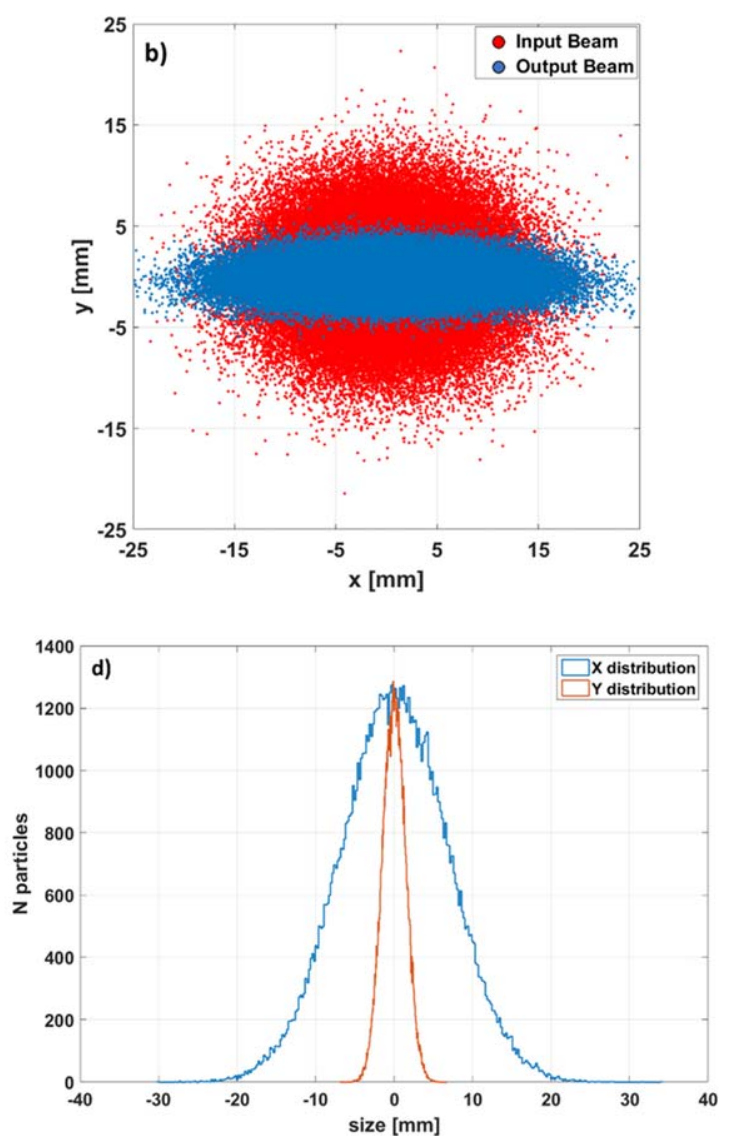

Figure 6. Particle distributions at the exit of the vector magnet (red) and at the isocenter (blue), resulting from the tracking of $10^{5}$ particles with Gaussian transverse distribution at 250 (a) and $70 \mathrm{MeV}$ (b). Beam profiles at isocenter for the 250 (c) and $70 \mathrm{MeV}$ (d).

Finally, figure 4(b) shows the decay of the magnetic field outside the toroidal magnet. In this case, the limit of the colour map is set at $50 \mathrm{mT}$ and this value is reached at $\mathbb{R}=180 \mathrm{~cm}$, i.e. about $30 \mathrm{~cm}$ outside the surface of the coils.

\section{D particle tracking}

The trajectories obtained as a result of the $2 \mathrm{D}$ tracking optimisation study, shown in figure 3 represent the reference orbits of the beams at each energy. To study the transmission of beams of finite transverse dimension we have used the 3D tracking algorithm described earlier. For each energy, a distribution of particle position and velocity as determined by the seeding procedure was generated at the location of the vector magnet. Since a broad spectrum of accelerators, i.e. cyclotrons, synchrotron or linacs, could be used to feed the gantry, a reference value of $\varepsilon_{1 \sigma}=1 \mathrm{~mm}$ mrad was selected as representative reference. Note that we indicate with $\varepsilon_{1 \sigma}$ the $1 \sigma$ normalised emittance.

The values of the Twiss parameters, $\alpha$ and $\beta$ were selected to obtain a round beam at high energies and they are listed in table 1 . The same parameters were kept for the whole spectrum of energies. 

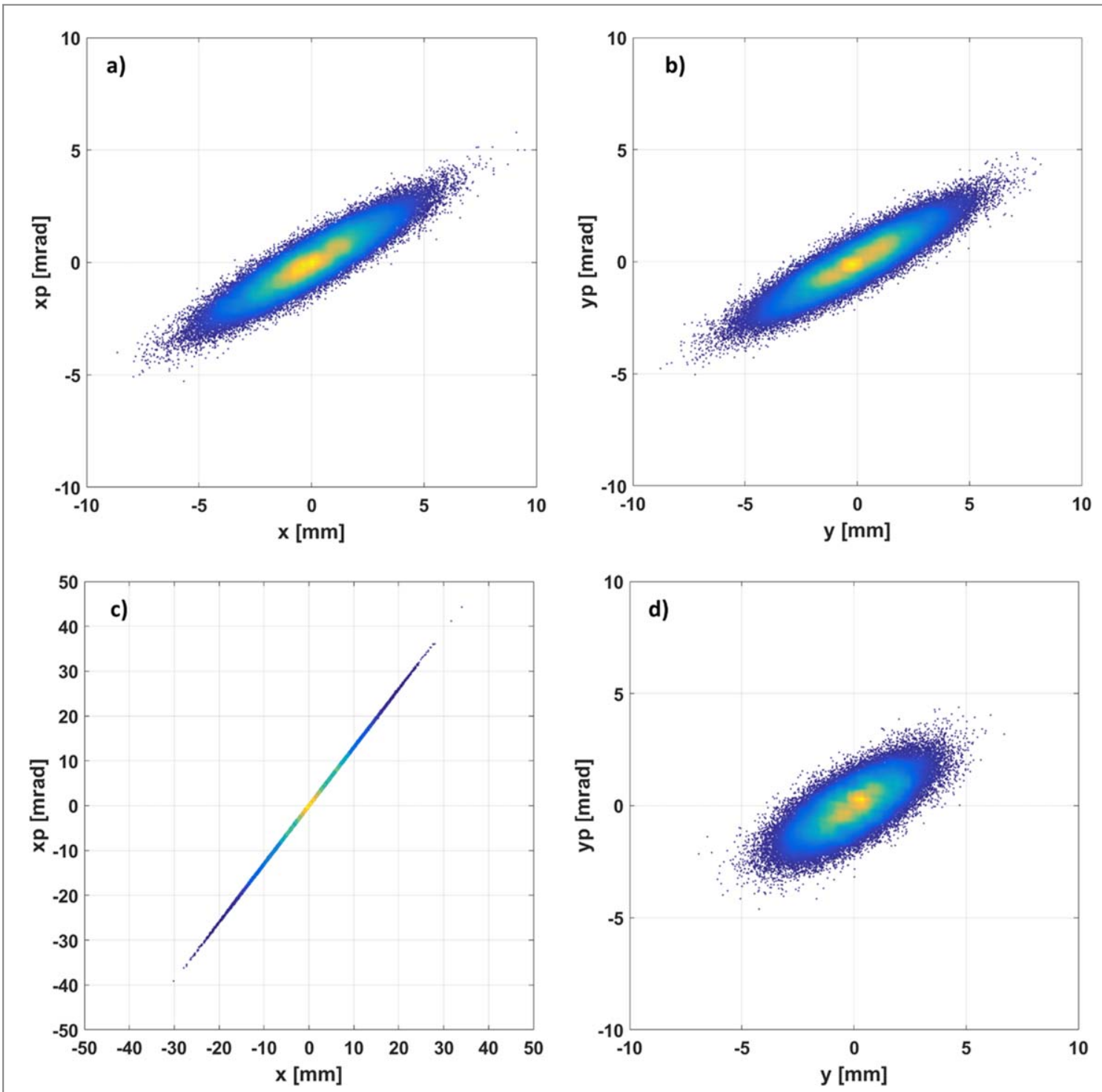

Figure 7. Horizontal and vertical phase space at isocenter, resulting from particle tracking at $250 \mathrm{MeV}$ (a), (b) and $70 \mathrm{MeV}$ (c), (d). The colour map indicates the density of particles.

Finally, a momentum spread $\Delta P / P$ equal to zero was assumed for the simulations reported below. This beam, consisting of $10^{5}$ particles, was then simulated, and the beam parameters at the isocenter were computed, using the definition of the covariance matrix of equation (8).

The trajectories of the $10^{5}$ particles tracked inside the GaToroid magnetic field for the energy of $250 \mathrm{MeV}$ are reported in figure 5 . The inset exhibits a zoom at the vector magnet location $\left(\mathbb{Z}=-z_{V}\right)$, showing the reference orbit (red line), coincident with the 2D tracking, and the reference plane (dashed line), where the input beam parameters were defined.

Figures 6(a) and (b) show the particle distributions at the vector magnet exit (in red), imposed as input, and at the isocenter (in blue), resulting from the multi-particle tracking for a beam energy of $250 \mathrm{MeV}$ and $70 \mathrm{MeV}$ respectively. Furthermore, figures 6(c) and (d) present the beam profiles in $x$ and $y$ for both energies. From these figures, it is evident that the choice of the Twiss parameters listed in table 1 allows obtaining a round beam at the isocenter at $250 \mathrm{MeV}$, while at $70 \mathrm{MeV}$ a severe asymmetry can be noted. Similar behaviour is shown in the phase spaces of figure 7, where the beam at $70 \mathrm{MeV}$ (figures 7(c) and (d)) became strongly divergent in the horizontal plane.

Using the fixed input parameters listed in table 1 , the three-dimensional particle tracking was repeated for different energies in the complete range of interest. The Twiss parameters at the isocenter were then computed, and the results are summarised in figure 8 . We see that at high energies the beam at the isocenter has a round shape. As the energy becomes lower, instead, the $\mathrm{x}$-dimension grows (increasing $\beta_{x}$ at decreasing energy), while it remains approximately constant in $y$ (constant $\beta_{y}$ ), creating an asymmetric beam profile. Applying the $\beta$-function definition, the beam envelope can be calculated as $E=\sqrt{\varepsilon_{1 \sigma} \beta}$ proportional to half of the width of 


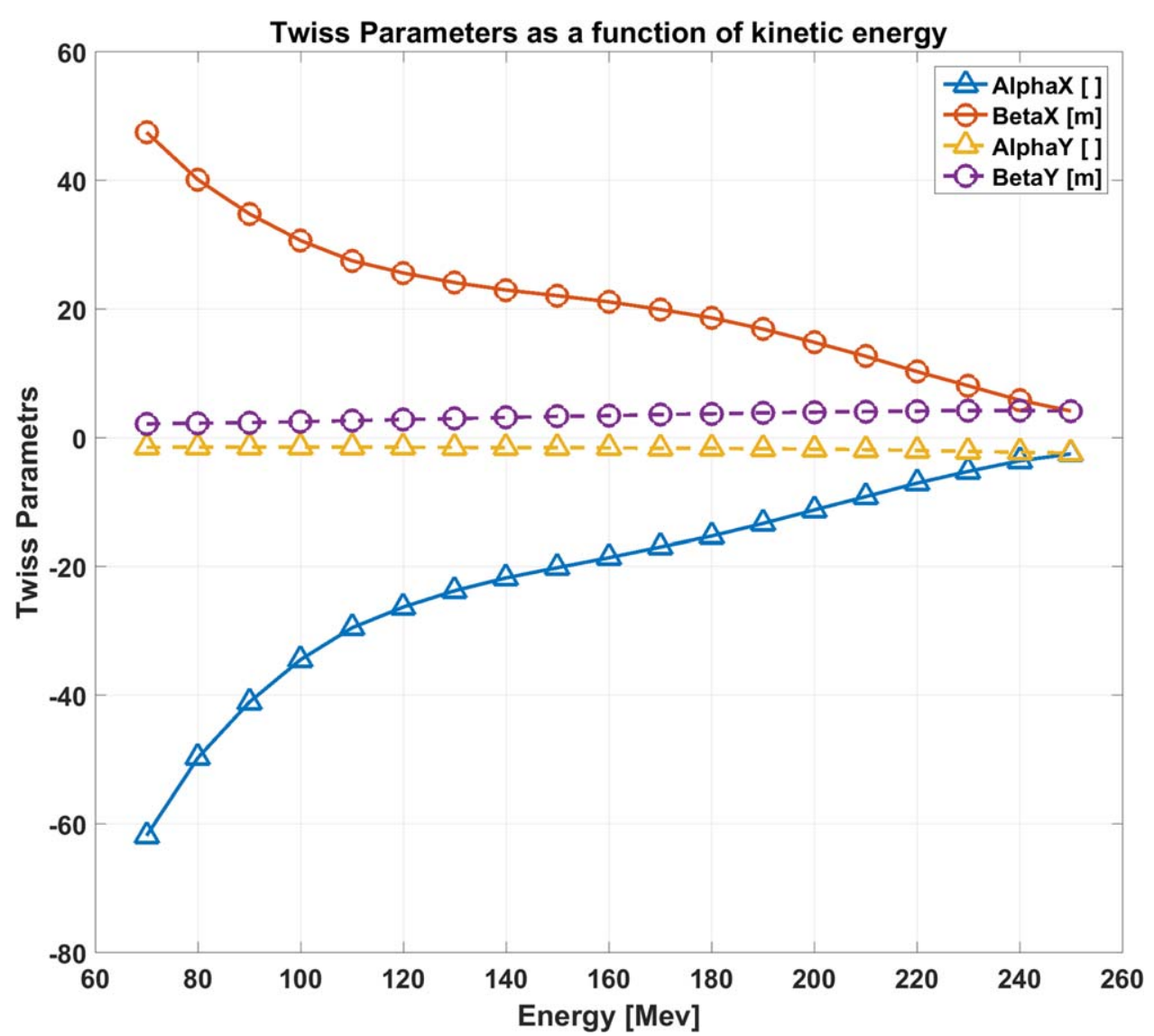

Figure 8. Twiss parameters as a function of beam kinetic energy.
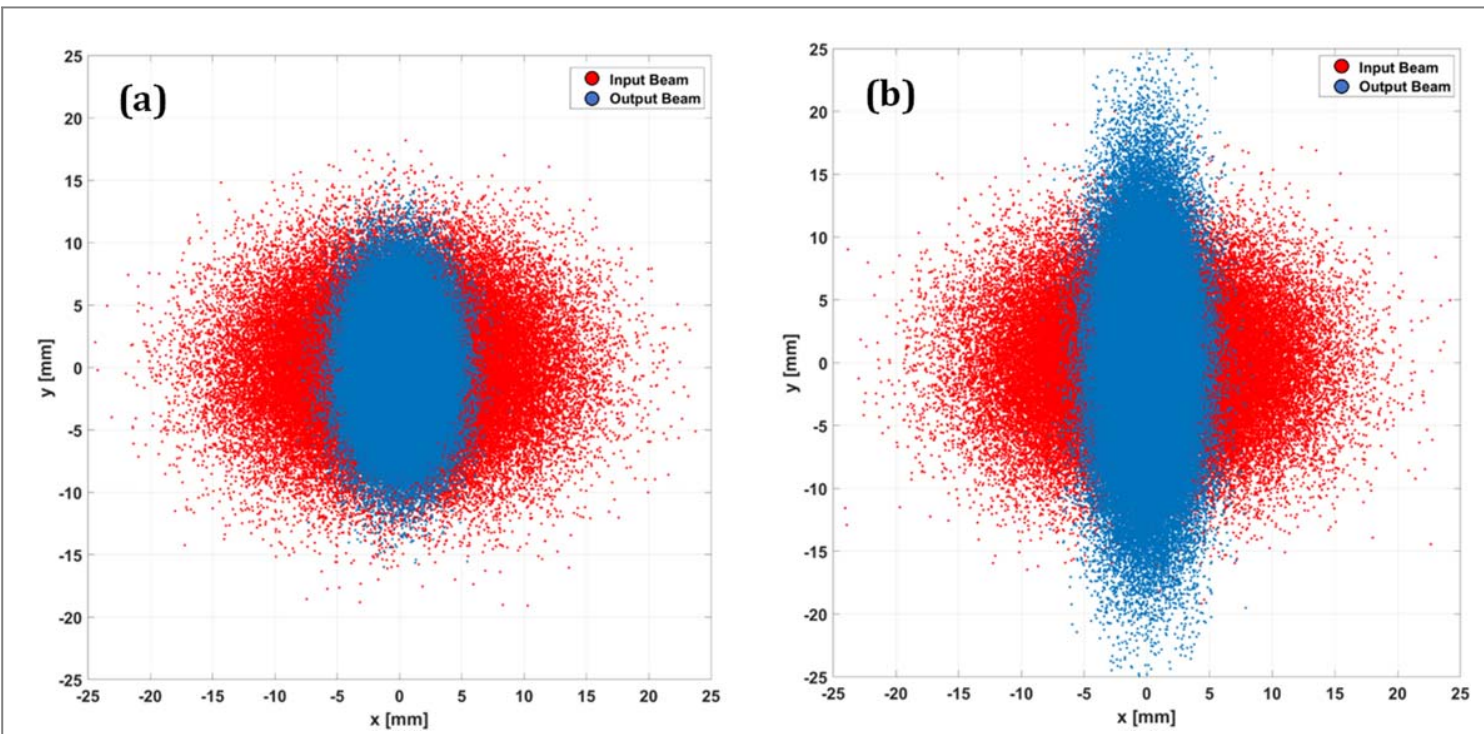

Figure 9. Particle distributions at the exit of the vector magnet (red) and at the isocenter (blue) for $d P / P=0.5 \%$ (a) and $d P / P=1 \%$ (b) at $250 \mathrm{MeV}$.

the Gaussian beam distribution at $1 \sigma$. At $250 \mathrm{MeV}$, the resulting beam envelope is $2.1 \mathrm{~mm}$ in both planes, while at $70 \mathrm{MeV}$ it is $5.5 \mathrm{~mm}$ and $1.5 \mathrm{~mm}$ in $x$ and $y$ respectively. Furthermore, as also shown in figure 8 , the values of $\alpha$ at isocenter indicate divergent beam in both planes for all the energies.

It is in principle possible to compensate for these effects by changing the input Twiss parameters, i.e. as done for the $250 \mathrm{MeV}$ energy. In practice, this would imply making use of a matching section (quadrupoles) placed upstream of the vector magnet. Since the design of the vector magnet assembly is only in an early stage of design, we did not proceed to an accurate matching procedure. Indeed, the results presented are mainly aiming to 
(a)

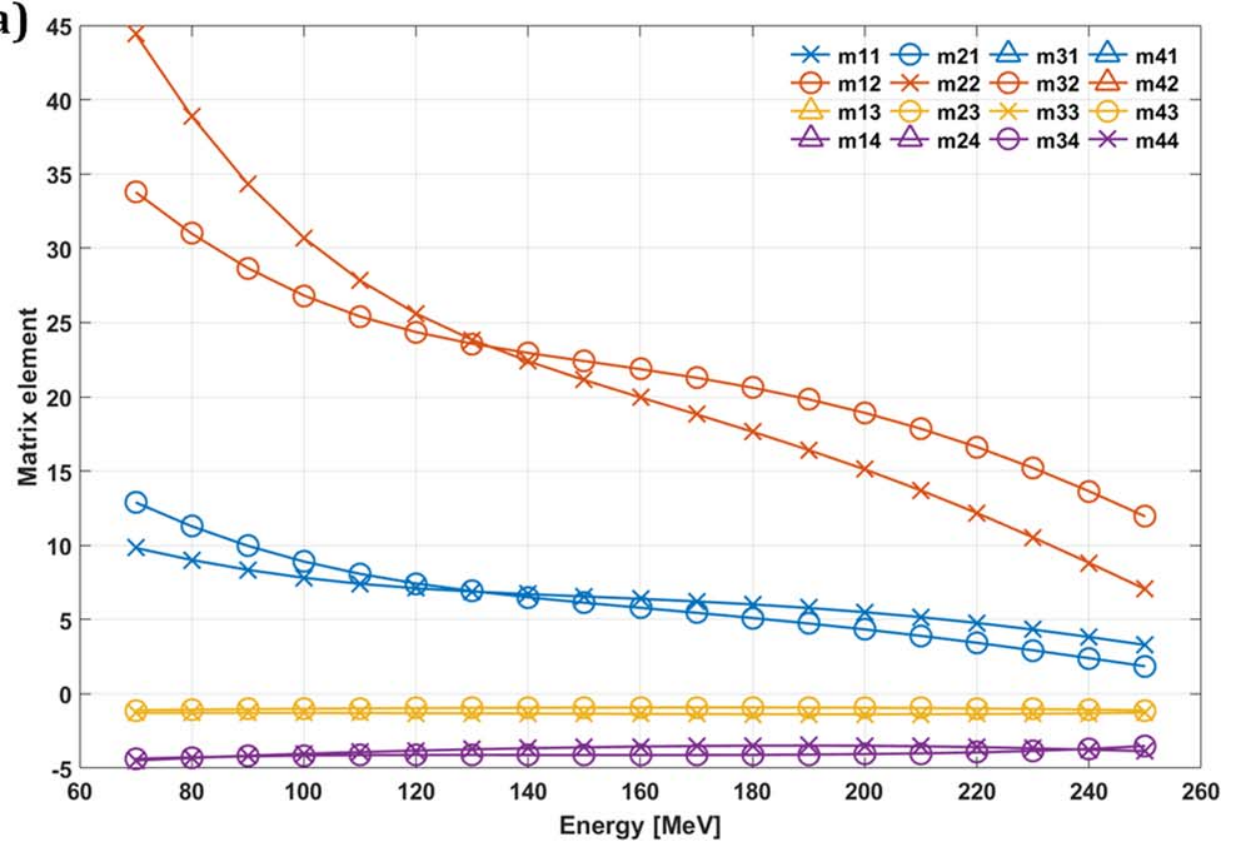

(b)

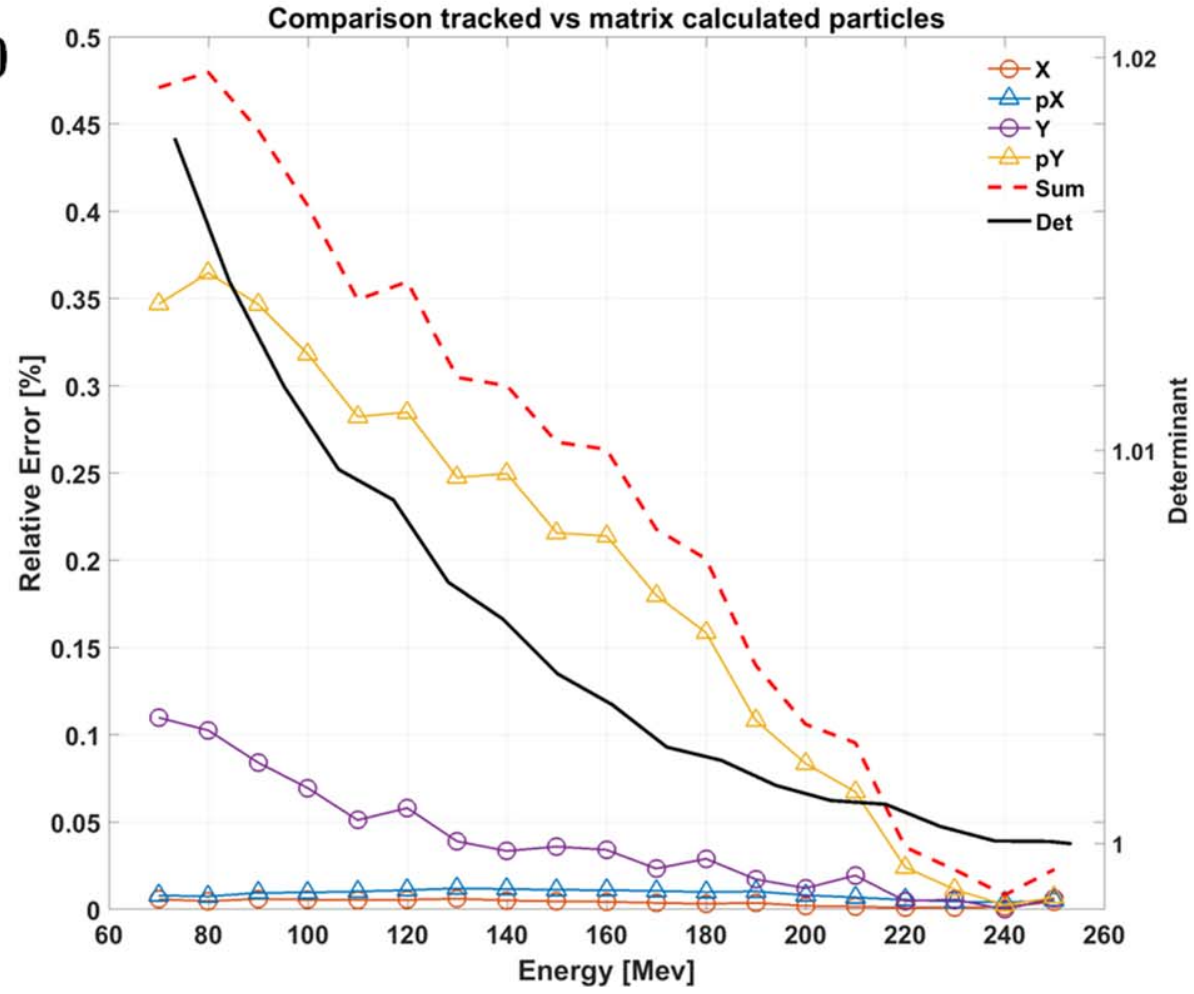

Figure 10. Transfer matrix element (a) and relative errors (b) as a function of beam energy. The sum of the errors is indicated in red dashed line, while the matrix determinant in black solid line (right axis).

demonstrate the validity of the developed three-dimensional particle tracking method between the end of the vector magnet and the isocenter, and the conceptual feasibility of the beam optics.

It is worth recalling that for low beam energies the multiple scattering in air and in the patient tissues becomes a dominating factor determining the beam spot size and its divergence (Garonna et al 2018). The results presented there highlight that no major difference can be identified between parallel, divergent or convergent beams in vacuum at isocenter within $\alpha= \pm 100$. The multiple scattering phenomenon almost completely overcomes the differences in beam size in the range quoted above.

In summary, the 3D tracking results, and considerations on multiple scattering show that the system has the potential to achieve beam properties at the isocenter that are coherent with clinical requirements, both in terms of beam size and divergence. 


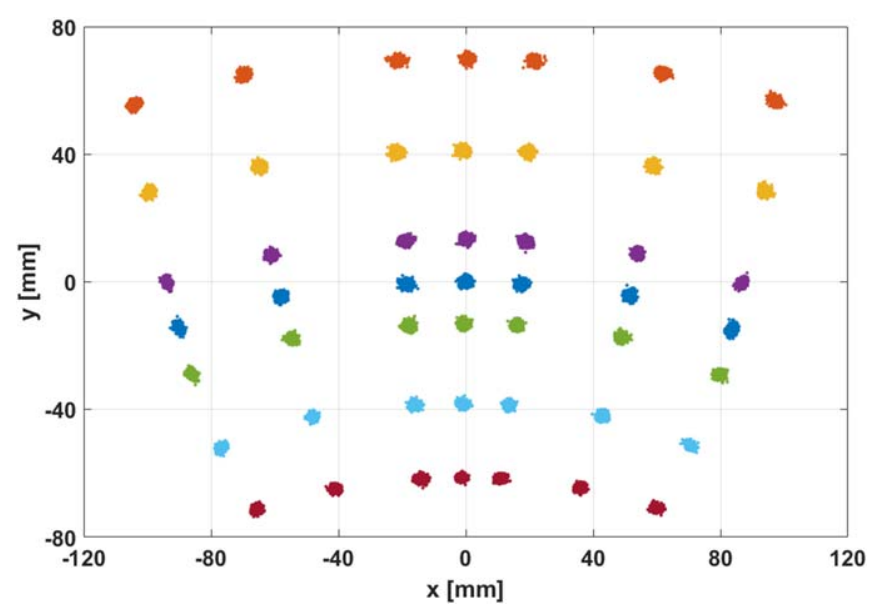

Figure 11. Natural response map of the beam scanning around the isocenter, for a linear variation of $\theta_{i}= \pm 0.4^{\circ}$ and $\alpha_{E}= \pm 1^{\circ}$ at 250 $\mathrm{MeV}$.

So far, we have assumed no momentum spread. However, given the long bending region inside the torus, the beam dispersion function $D$ grows naturally, increasing the beam dimension on the bending plane $(y)$ by a factor $D \frac{d P}{P}$.

This effect can be appreciated in the results of figure 9 where we have assumed an energy of $250 \mathrm{MeV}$ and two Gaussian distributions of momentum spread of $0.5 \%$ and $1 \%$. In this analysis, the effect of the energy spread is produced exclusively by the beam path inside the gantry, as the dispersion is assumed to be zero at the exit of the vector magnet. Again, this effect could be anticipated by modifying the dispersion function at the vector magnet location, which would affect the particle trajectories in the gantry, as well as the beam size. Without simulations of a complete beamline, however, it is not trivial to assess the real effect of the energy spread on the beam at isocenter. As introduced in Bottura (2018) Bottura et al (2020), a possible alternative solution could imply the use of double achromatic torus, as adopted by Boyd et al (1973), Bromberg and Michael (2017). Given the large aperture required to accommodate all the beam orbits, another option could be to propagate a non-zero dispersion from the vector magnet through the gantry, designing the focusing such that the dispersion is suppressed at the isocenter. These ideas are not investigated further in this paper.

\section{Transfer matrices}

As outlined earlier, the results of 3D tracking can be used to solve equation (14) and evaluate the linear transfer matrices of the system. The benefits of a linear transfer matrix are evident, especially the possibility to greatly simplify the calculation of the beam properties at the isocenter without the burden of a full 3D tracking. The terms of the transfer matrices have been evaluated as a function of energy using the 3D multi-particle tracking between 70 and $250 \mathrm{MeV}$. The result is reported in figure 10(a) where we plot the matrices elements as a function of beam energy. Even if the values of the elements are not trivial to interpret, the trends provide useful insights to understand the physics of the system. First of all, the coupling terms (the elements of the $2 \times 2$ off-diagonal blocks) are constant and very close to zero for all energies. This means that the coupling between $x$ - and $y$-planes is negligible, and the two planes can be treated separately with good approximation. Furthermore, it is possible to observe a monotonic reduction in modulus of the diagonal elements, more evident on the $x$-plane, i.e. $m_{11}, m_{12}, m_{21}, m_{22}$. This reduction is coherent with the trend obtained for the Twiss parameters plotted in figure 8. Finally, it is useful to note that the behaviour is rather regular, with no discontinuity, which could be amenable to an accurate interpolation strategy.

In figure 10(b) we have reported the relative errors $\lambda$ for the beam positions $x, y$, and respective derivatives $x^{\prime}, y^{\prime}$, as defined by equation (15). This plot shows the quality of the matrix representation when compared to $3 \mathrm{D}$ tracking. As the errors tend to increase as the kinetic energy decreases, the results also point to the sensitivity of low rigidity beam to magnetic field perturbations. The errors on the bending plane, $\lambda_{y}$ and $\lambda_{y^{\prime}}$, are significantly higher than the others, but still below $0.1 \%$. The red dashed line indicates the sum of the four $\lambda$ relative errors, which is at most $0.15 \%$.

Finally, we also plot in figure 10(b) the determinant of the extrapolated matrices. As previously explained, in the theoretical case the matrix has unit determinant. Coherently with the $\lambda$ errors, the determinant deviates from the unity al low energies, with a maximum error of less than $2 \%$. 


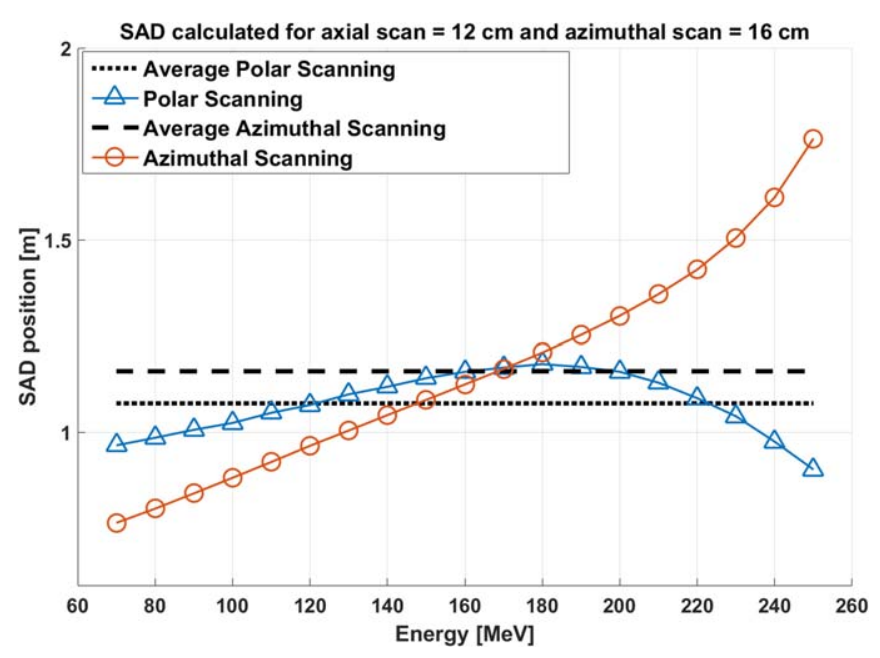

Figure 12. Effective source-to-axe distance (SAD) as a function of energy for axial (blue triangles) and transverse (red circles) scanning.

\section{Scanning system}

The tracking simulations described above were performed for nominal input angles at the vector magnet, $\theta_{i}$ and $\alpha_{E}$. Any deviation from the nominal angles results in a change in the beam position around the isocenter. Specifically, variations of $\alpha_{E}$ result in a movement of the beam along the $\mathbb{Z}$-axis, while variations of $\theta_{i}$ yield a transverse movement, normal to the $\mathbb{Z}$-axis. This feature can be used to perform pencil beam scanning, moving the beam into a defined scanning area around the isocenter. Referring to the description of the general concept of GaToroid, this can be achieved by modulating the kick of the vector magnet, which can be used as an upstream scanning system. As previously explained, and without entering in the details of the vector magnet design, the different magnitudes (radian versus milliradian) and timescales (seconds versus milliseconds) between setting the polar/azimuthal direction and setting the scanning angles probably require different systems for the two functions.

For the system considered here, figure 11 shows the natural response map of the beam scanning in an area of about $20 \mathrm{~cm} \times 15 \mathrm{~cm}$ for the $250 \mathrm{MeV}$ case, resulting from a linear angle deviation of $\alpha_{E}= \pm 1^{\circ}$ and $\theta_{i}= \pm 0.4$ . A variation of the kick in the polar angle of $\alpha_{E}= \pm 1^{\circ}$ is translated into $\pm 60 \mathrm{~mm}$ along the axial direction of the torus. An azimuthal deviation of $\theta_{i}= \pm 0.4^{\circ}$ corresponds to a scanning of $\pm 50 \mathrm{~mm}$ in the gantry azimuthal direction. The shape of the scanning area can be adjusted acting on the angle of the vector magnet, to contour the treatment region. It is important to underline that these results indicate also the accuracy required at the vector magnet to obtain the precise beam positioning at the isocenter. To maintain the beam position at the isocenter within $1 \mathrm{~mm}$, the precision required at the vector magnet is in the order of $0.01^{\circ}$ (few $\mathrm{mrad}$ ). This constraint may pose a relevant challenge in the design of the vector magnet.

The area is azimuthally limited by the geometrical aperture available between the coils, that for this 16 coils proton GaToroid is about $10 \mathrm{~cm}$. Common values for the required minimal size of the treatment field at the isocenter are about $20 \mathrm{~cm} \times 20 \mathrm{~cm}$, and are driven by the intent to improve the treatment quality and reduce the field overlaps. The results shown are intended to be a proof of principle for the system scanning feature. To provide wider scanning field size, especially in the azimuthal direction, topological modifications are required in the gantry, i.e. larger bore, reduced number of coils or modified angular periodicity of the coils (i.e. what we have referred to as parallel channel configuration Bottura et al 2020).

A quantity that is of high relevance for the therapy quality is the effective source-to-axis distance (SAD), namely the distance between the virtual point source and the local position of the Brag peak at the isocenter. The source of the beam kick is the vector magnet, but the beam bending in the toroidal field results in a virtual source point located at some distance from the patient. We identify this virtual source point using the beam incidence position and angle on the torus axis, finding the virtual origin of the cone of straight trajectories.

Figure 12 shows the effective SAD distance as a function of the beam kinetic energy, for both axial (acting on $\alpha_{E}$ ) and transverse scanning (acting on $\theta_{i}$ ). The average SADs for axial and transverse scanning are in the order of $1 \mathrm{~m}$. The transverse scanning is monotonically increasing with the energy given the fact that beams with higher momentum are entering the torus in a larger radial position. On the other side, the trend of the axial SAD is flatter and determined by the field profile. The actual value of SAD is similar to the one of the downstream scanning gantries, typical in the order of $1-2 \mathrm{~m}$, and does not provide a parallel scanning.

While promising, we see that the configuration studied will require improvement. In particular, a SAD of the order of $4 \mathrm{~m}$ is the required minimum to remain within a maximum threshold of $20 \%$ of surface dose increase 
Table 2. Comparison of the main features of the presented GaToroid configuration with state-of-the-art gantries for protons.

\begin{tabular}{lcc}
\hline Parameter & GaToroid & Rotational gantries \\
\hline Diameter & $3.3 \mathrm{~m}$ & $7-10 \mathrm{~m}$ \\
Weight & 12 tons & $110-270$ tons \\
Irradiation directions & 16 & all \\
Electromagnetic operation & Steady-state & Transient \\
Mechanical operation & Steady-state & Rotation \\
Vacuum & Tailored & Standard \\
Cryogenics & Tailored & Standard \\
Clinical practise and TPS & Tailored & Standard \\
\hline
\end{tabular}

(Pullia et al 2009). Furthermore, though not a limitation of the system, present commercial treatment planning systems (TPS) usually work with a constant SAD in the complete energy range. Both requirements could be approached by modifying the field profile at the exit of the magnet to increase the $S A D$, shaping the pole face exit angle of the coils to obtain parallel scanning (i.e. infinite SAD) and negligible beam energy dependence (Koschik et al 2012, Osorio et al 2012). A further geometrical optimisation could be performed on the coil exit region, without affecting the validity of the results obtained so far.

\section{General discussion}

Given the novelty of a GaToroid machine, the analysis and optimisation require different approaches compared to the traditional gantries for hadron therapy.

First of all, differently from traditional transfer lines and gantries, the main orbit, around which the linear beam optics formalism is usually applied, is not fixed by the construction of the machine and is dependant on beam kinetic energy. This dependence is at the basis of a GaToroid working principle and cannot be avoided, but the torus magnetic field can be optimised to have all orbits coincident at the isocenter. In the presented solutions, the difference of the orbit position at the isocenter is less than one millimetre. We considered this value of precision coherent with the beam positioning requirements of the traditional rotational gantries. Once the orbits have been calculated, it is possible to evaluate the effect of the toroidal field on a beam. Also in this case, traditional tools that use a series of transfer matrix to represent and optimise the system cannot be used. Since at the moment a formalism to describe the GaToroid magnetic field in terms of bending and focusing elements is still missing, we proceeded with multi-particle tracking. The procedure highlights how GaToroid behaves like a combined function magnet, presenting quadrupolar gradients in addition to the main dipole field. The lack of active focusing elements uncoupled with the bending field could represent a challenge for the operation of the machine. At the same time, the long drift space between the torus and the vector magnet makes the system very sensitive to misalignments and orbit deviation. In the present design, the presence of beam monitors and correctors have been neglected, but they clearly need to be taken into account for the effective functioning of the machine. Despite the study of a GaToroid machine is still in its infancy, it is worth underlining the potential benefits and drawbacks of this concept when compared to traditional rotational gantries. As summarised in table 2, the GaToroid concept has the potential to strongly reduce size and weight; this comes with the price of a reduced number of irradiation directions. If on the one hand, the torus operates in steady-state both mechanically and electrically, on the other hand, it would require tailored systems for vacuum and cryogenics. Finally, specific features of GaToroid, such as the discrete number of irradiation directions, limited size of the bore and SAD variation with energy, need to be thoroughly investigated and implemented in clinical practises and TPSs.

\section{Conclusions}

We have presented in this paper an analysis of the beam transfer properties of the static toroidal gantry GaToroid. A 2D particle tracking, integrated with magnetic field calculation, was developed and used in an optimisation algorithm with the goal of creating a sufficiently large acceptance magnet. The results show that the beam orbits in the complete treatment energy range can be directed to the isocenter within a precision of $1 \mathrm{~mm}$, confirming the basic principle of GaToroid.

A 3D linear beam motion formalism was then developed and used to perform particle tracking around the orbits calculated in 2D. Thanks to this analysis, we were able to determine the beam optics characteristics of the toroidal field and to show the sensitivity of the beam position at the isocenter when small kicks are applied 
upstream of the toroid. The main result of these studies is that the beam parameters obtained at the isocenter for a beam with negligible momentum spread are compatible with clinical requirements. Also, the upstream kick can be used to effectively 'paint' a spot of relevant dimension at the isocenter location.

At the same time, the analysis shows that more work is required. Beam matching is one area of study, to compensate for the de-focusing observed, as well as the large dispersion generated by the long bending region inside the gantry. Moreover, the scanning field size needs to be enlarged to perform high-quality treatments, and the SAD needs an increase by a factor two to three. This can be done adapting the gantry topology and further optimising the pole face angle at the exit of the coils. Finally, we have shown how to identify a linear transfer matrix that provides a good model of the variation of the beam characteristics (position and derivative) through the toroidal field. The matrix represents precisely the global parameters of a beam of a given energy, and can be easily obtained for any energy of interest. Such matrices can be used to greatly simplify the integration with traditional beam optics systems and codes, e.g. to perform a matching between the extraction from the accelerator and the vector magnet. The proposed work appears to us very promising, and the presented methodology is an initial solid basis towards the development of a new generation of gantries for protons, as well as heavier ions, such as helium and carbon.

\section{Acknowledgments}

The authors wish to acknowledge the advice and the stimulating discussions on theoretical beam optics and more practical numerical solutions with Andrea Latina and Vittorio Bencini (CERN), as well as Marco Pullia and Simone Savazzi (CNAO).

\section{ORCID iDs}

E Felcini (iD https:// orcid.org/0000-0001-8965-7196

\section{References}

Andreev V V and Yudin I P 1998 Calculation of high-order beam optics in toroidal magnetic lens Proc., 17th Int. Conf. on High-Energy Accelerators, HEACC 19981998 (Dubna, Russian Federation, 07-12 September) 199-201

Beatson R and Greengard L 1997 A short course on fast multipole methods Wavelets, Multilevel Methods and Elliptic PDEs (Numerical Mathematics \& Scientific Computation) ed M Ainsworth, J Levesley, W Light and M Marletta (New York: Oxford University Press) $1-37$

Bottura L 2018 A gantry and apparatus for focussing beams of charged particles WO2019/224215https:/ worldwide.espacenet.com/ patent/search/family/062235811/publication/WO2019224215A1?q=pn\%3DEP3573075A1

Bottura L, Felcini E, Ferrero V, Fiorina E, Monaco V, Pennazio F, de Rijk G and Cerello P 2020 Delivery, beam and range monitoring in particle therapy in a highly innovative integrated design Front. Phys. Med. Phys. Imaging 8544

Bottura L, Felcini E, De Rijk G and Dutoit B 2020 GaToroid: a novel toroidal gantry for hadron therapy Nucl. Instrum. Methods Phys. Res. A 983164588

Boyd D, Schwettman H A and Simpson J 1973 Large acceptance pion channel for cancer therapy Nucl. Instrum. Methods 111 315-31

Bromberg L and Michael P C 2017 Toroidal bending magnets for hadron therapy gantries US9711254B2https://patents.google.com/ patent/US9711254

Brouwer L, Caspi S, Robin D and Wan W 2013 3D toroidal field multipoles for curved accelerator magnets Proc. PAC2013 (CA, USA) 907-9 https://escholarship.org/uc/item/4z161518

Durante J S L M and Orecchia R 2017 Charged-particle therapy in cancer: clinical uses and future perspectives Nat. Rev. Clin. Oncol. 14 483-95

Farr J B, Flanz J B, Gerbershagen A and Moyers M F 2018 New horizons in particle therapy systems Med. Phys. 45 953-83

Felcini E, Harray J, Lehtinen T, Perini D, Perez J C, de Rijk G and Dutoit B 2021 Design of the first HTS single-coil demonstrator of GaToroid toroidal gantry for hadron therapy IEEE Trans. Appl. Supercond. 31 1-5 4400205

Felcini E 2021 Analysis of a novel toroidal configuration for hadron therapy gantries École Polytech. fédérale Lausanne EPFL https:// infoscience.epfl.ch/record/283295

Felcini E, Bottura L, van Nugteren J, de Rijk G, Kirby G and Dutoit B 2020 Magnetic design of a superconducting toroidal gantry for hadron therapy IEEE Trans. Appl. Supercond. 30 1-5 4400405

Ferrario M, Migliorati M and Palumbo L 2013 Space charge effects Proc. CAS-CERNAccel. Sch. Adv. Accel. Phys. 009331-56

Fuchs R, Weinrich U and Emde P 2004 The heavy ion gantry of the HICAT facility Proc. EPAC 2004, (Lucerne, Switz.)

Gambini L, Breschi M, Felcini E, Cristofolini A and Bottura L 2020 An algorithm for toroidal field harmonics computa- tion in arbitrary magnetic configurations IEEE Trans. Appl. Supercond. 30 1-5

Garonna A et al 2018 Beam parameters optimization and characterization for a turning linac for protontherapy Phys. Med. 54 152-65

Gerbershagen A, Meer D, Schippers J M and Seidel M 2016 A novel beam optics concept in a particle therapy gantry utilizing the advantages of superconducting magnets Zeitschrift für Medizinische Physik 26 224-37

Golub G H and Van Loan C F 1996 Matrix Computations 3rd edn (London: The Johns Hopkins University Press) pp 498

Greengard L F 1987 The Rapid Evaluation of Potential Fields in Particle Systems (New Haven, CT: Yale University Press)

Hadron M et al 2014 The Pro. Nova SC360 Gantry vol 26 (Daresbury, UK: Cockcroft Institute) pp 3-8

Iwata Y et al 2012 Design of a superconducting rotating gantry for heavy-ion therapy Phys. Rev. ST Accel. Beams 15044701 
Iwata Y, Shirai T and Noda K 2016 Design of superconducting magnets for a compact carbon gantry IEEE Trans. Appl. Supercond. 26 4400104

Kang M and Pang D 2020 Commissioning and beam characterization of the first gantry-mounted accelerator pencil beam scanning proton system Med. Phys. 47 3496-510

Kim J and Yoon M 2019 Design of a compact gantry for carbon-ion beam therapy Phys. Rev. Accel. Beams 22101601

Koschik A, Bula C, Duppich J, Gerbershagen A, Grossmann M, Schippers J M and Welte J 2015 Gantry 3: further development of the PSI Proscan proton therapy facility 6th Int. Part. Accel. Conf. IPAC2015 (Richmond, VA, May 3-8, 2015) (https://doi.org/10.18429/ JACoW-IPAC2015-TUPWI016)

Koschik A, Dorda U, Meer D and Pedroni E 2012 The medaustron proton gantry IPAC 2012_Int. Part. Accel. Conf. 2012 (New Orleans, USA, May, 2-25, 2012) 4091-3

Kraft G 1990 The radiobiological and physical basis for radiotherapy with protons and heavier ions Strahlenther. Onkol. 166 10-3

Kumata Y 2013 SUMITOMO particle therapy technologies 55th AAPM Annu. Meet. Beam Ther. Symp. August 3, 2013

Kyng T J and Konstandatos O 2014 Multivariate Monte-Carlo simulation and economic valuation of complex financial contracts: an excel based implementation Spreadsheets Educ. 7 1-38

Lawson J and Tigner M 1984 The Physics of Particle Accelerators Ann. Rev. Nucl. Part. Sci. 34 99-123

La Civita S 2019 Advances in integrated compactsingle-room proton therapysystems, ENLIGHT annual meeting 2019 (Caen, France, July 3rd) https://indico.cern.ch/event/783037/contributions/3456742/attachments/1882018/3101261/Enlight_1.pdf

Masood U et al 2017 A light-weight compact proton gantry design with a novel dose delivery system for broad-energetic laser-accelerated beams Phys. Med. Biol. $625531-55$

Mohri A, Ikuta K and Fujita J 1977 New lens system using toroidal magnetic field for intense ion beam Japan. J. Appl. Phys. 16 491-6

Myers S, Degiovanni A and Farr J B 2019 Future prospects for particle therapy accelerators Rev. Accel Sci. Technol. 10 49-92

Nesteruk K P, Bolsi A, Lomax A J, Meer D, van de Water S and Schippers J M 2021 A static beam delivery device for fast scanning proton arctherapy Phys. Med. Biol. 66055018

Osorio J, Priano C and Pullia M 2012 Electromagnetic characterization of a big aperture magnet used in particle beam cancer(ECBAMUPBC) Proc. COMSOL Conf. (Milan)

Pearson E, de Wilde O, Doyen R, Forton E, Jongen Y, Krier G, Neuvéglise D and Zaremba S 2014 Magnet developments and commissioning for the IBA compact gantry IEEE Trans. Appl. Supercond. 244401004

Pedroni E, Meer D, Bulla C, Safai S and Zenklusen S 2011 Pencil beam characteristics of the next-generation proton scanning gantry of PSI: design issues and initial commissioning results Eur. Phys. J. Plus 12666

Lante V et al 2012 Conceptual design for a carbon ion gantry - ULICE Report no: JRA 6.3 ULICE (https://doi.org/10.13140/2.1.1930.1445)

Robin D S et al 2011 Superconducting toroidal combined-function magnet for a compact ionbeam cancer therapy gantry Nucl. Instruments Methods Phys. Res. A 659 484-93

Schippers J M, Lomax A, Garonna A and Parodi K 2018 Can technological improvements reduce the cost of proton radiation therapy? Semin. Radiat. Oncol. 28 150-9

Symonds P, Mills J A and Duxbury A 2019 Walter and Miller's Textbook of Radiotherapy: Radiation Physics, Therapy and Oncology 8th edn (Amsterdam: Elsevier)

Takada K 2020 Qualitative comparison of proton beams and other radiotherapy beams ed K Tsuboi et al Proton Beam Radiotherapy (Singapore: Springer Nature)

Trbojevic D, Parker B, Keil E and Sessler A M 2007 Carbon/proton therapy: a novel gantry design Phys. Rev. Spec. Top.-Accel. Beams 10 1-6

Tsujii H et al 2007 Clinical results of carbon ion radiotherapy at NIRS J. Radiat. Res. 48 A1-13

Tsujii H, Kamada T, Shirai T, Noda K, Tsuji H and Karasawa K 2013 Carbon-Ion Radiotherapy: Principles, Practices, and Treatment Planning. (Berlin: Springer)

Umezawa M 2014 Hitachi proton beam therapy therapy system with various types of rotating gantries Modern Hadron Therapy Gantry Developments (Warrington: Cockcroft Institute)

Von Essen C F et al 1982 The piotron: initial performance, preparation and experience with pion therapy Int. J. Radiat. Oncol. Biol. Phys. 8 1499-509

van Milligen B P and Lopez Fraguas A 1994 Expansion of vacuum magnetic fields in toroidal harmonics Comput. Phys. Commun. 81 74-90

van Nugteren $\mathrm{J} 2011$ Software development for the science and design behind superconducting magnet systems Cern Internsh. Report 2011 EDMS Report No: 2480485 CERN https://edms.cern.ch/document/2480485/1

van Nugteren J 2016 High temperature superconductor accelerator magnets University of Twente, The Netherlands https:/ / research.utwente. $\mathrm{nl} / \mathrm{en} /$ publications/high-temperature-superconductor-accelerator-magnets

Van Nugteren J 2019 RAT software Cern TE-MSC-MDT Eng. Meet https://indico.cern.ch/event/856850/contributions/3606658/ attachments/1944535/3225730/EngineeringMeeting_14_11_2019_Jvn_v1.pdf

Yan S, Lu H-M, Flanz J, Adams J, Trofimov A and Bortfeld T 2016 Reassessment of the necessity of the proton gantry: analysis of beam orientations from 4332 treatments at the Massachusetts general hospital proton center over the past 10 years Int. J. Radiat. Oncol. 95 224-33

Yang W Y, Cao W, Kim J, Park K W, Park H-H, Joung J, Ro J S, Lee H L, Hong C H and Im T 2020 Applied Numerical Methods Using MATLAB 2 edn (New York: Wiley) https:/ /www.mathworks.com/academia/books/applied-numerical-methods-using-matlabyang.html

Wan W, Brouwer L, Caspi S, Prestemon S, Gerbershagen A, Schippers J M and Robin D 2015 Alternating-gradient canted cosine theta superconducting magnetsfor future compact proton gantries Phys. Rev. ST Accel. Beams 1810350 